\title{
Severe COVID-19 in Uganda across Two Epidemic Phases: A Prospective Cohort Study
}

Barnabas Bakamutumaho, ${ }^{1}$ Matthew J. Cummings, ${ }^{2,3 \star}$ Nicholas Owor,${ }^{1}$ John Kayiwa, ${ }^{1}$ Joyce Namulondo, ${ }^{1}$ Timothy Byaruhanga, ${ }^{1}$ Moses Muwanga, ${ }^{4}$ Christopher Nsereko, ${ }^{4}$ Emmanuel Rwamutwe, ${ }^{4}$ Roselyn Mutonyi, ${ }^{4}$ Josephine Achan, ${ }^{4}$ Lucy Wanyenze, ${ }^{4}$ Alice Ndazarwe, ${ }^{4}$ Ruth Nakanjako, ${ }^{4}$ Richard Natuhwera, ${ }^{4}$ Annet Nsangi, ${ }^{4}$ Henry Kyobe Bosa, ${ }^{1,5}$ Felix Ocom, ${ }^{6}$ Max R. O'Donnell, ${ }^{2,3}$ Bernard Kikaire, ${ }^{1,7}$ and Julius J. Lutwama, ${ }^{1}$ Collaboration for Clinical and Laboratory Characterization of COVID-19 in Uganda

${ }^{1}$ Uganda Virus Research Institute, Entebbe, Uganda; ${ }^{2}$ Vagelos College of Physicians and Surgeons, Columbia University, New York, New York; ${ }^{3}$ Mailman School of Public Health, Columbia University, New York, New York; ${ }^{4}$ Entebbe Regional Referral Hospital, Entebbe, Uganda; ${ }^{5}$ Uganda Peoples' Defence Force, Kampala, Uganda; ${ }^{6}$ Ministry of Health, Kampala, Uganda; ${ }^{7}$ Makerere University College of Health Sciences, Kampala, Uganda

\begin{abstract}
Among a prospective cohort of children and adults admitted to a national COVID-19 treatment unit in Uganda from March to December 2020, we characterized the epidemiology of and risk factors for severe illness. Across two epidemic phases differentiated by varying levels of community transmission, the proportion of patients admitted with WHO-defined severe COVID-19 ranged from 5\% (7/146; 95\% Cl: 2-10) to 33\% (41/124; 95\% Cl: 25-42); 21\% (26/124; $95 \% \mathrm{Cl}: 14-29 \%$ ) of patients admitted during the peak phase received oxygen therapy. Severe COVID-19 was associated with older age, male sex, and longer duration of illness before admission. Coinfection with HIV was not associated with illness severity; malaria or tuberculosis coinfection was rare. No patients died during admission. Despite low mortality, hospital incidence of severe COVID-19 during the first epidemic peak in Uganda was substantial. Improvements in vaccine deployment and acute care capacity, including oxygen delivery, are urgently needed to prevent and manage severe COVID-19 in sub-Saharan Africa.
\end{abstract}

\section{INTRODUCTION}

Little is known about the clinical epidemiology of severe COVID-19 in sub-Saharan Africa (SSA). In Uganda, where SARS-CoV-2 was imported via travel in March 2020, over 80,000 case have been reported. ${ }^{1-3}$ Similar to other settings, distinct epidemic phases of COVID-19 have been observed in Uganda, differentiated by scenarios of sporadic cases followed by community transmission and hospital surges. ${ }^{4}$ Here, we characterize the epidemiology of and risk factors for severe COVID-19 among a prospective cohort of children and adults admitted to a national COVID-19 treatment unit in Uganda during the first two phases of the epidemic.

\section{METHODS}

This prospective observational study was conducted at Entebbe Regional Referral Hospital (ERRH), a 200-bed public hospital in central Uganda, from March 22 to December 31, 2020. During this period, ERRH was designated as a national isolation and treatment unit; only patients with COVID-19 were admitted to the unit. There is no intensive care unit or piped oxygen available at ERRH. Oxygen concentrators with capacity to provide 4-6 L/minute of oxygen, typically via nasal cannula, were provided as part of the study program.

Patients were included in this study if they fulfilled the following criteria: 1) were admitted to the ERRH COVID-19 treatment unit during the study period, 2) $\geq 5$ years of age, 3) had laboratory-confirmed SARS-CoV-2 infection, and 4) were able to provide informed consent or had a surrogate, parent, or guardian available to do so. Pregnant women were excluded. Testing for SARS-CoV-2 was performed on nasoand/or oro-pharyngeal swab samples at Uganda Virus Research Institute or other Ministry of Health-certified testing

*Address correspondence to Matthew J. Cummings, Division of Pulmonary, Allergy, and Critical Care Medicine, Columbia University Irving Medical Center, 622 West 168th St., PH 8E-101, New York, NY 10032. E-mail: mjc2244@columbia.edu centers using polymerase chain reaction (Charité, Berlin, Germany; DaAnGene Co. Ltd, Guangzhou, China).

At enrollment, clinical assessments were performed, and data were recorded using a modified form developed by the International Severe Acute Respiratory and Emerging Infection Consortium and WHO. For all patients, rapid testing was performed for malaria and HIV. For HIV-infected patients, testing for tuberculosis (TB) was performed if samples were obtainable. Details on pathogen diagnostics are provided in the supplemental materials.

We divided the study period into two phases because criteria for admission to the COVID-19 unit at ERRH, defined by Ministry of Health guidelines, were modified over time in response to evolving case surges. ${ }^{5}$ These guidelines set thresholds for admission based on bed surge capacity across isolation and treatment units nationwide. ${ }^{5}$ When $\geq 60 \%$ of bed capacity dedicated to COVID-19 management was available (approximately March-July 2020, a period with more sporadic cases and less community transmission; study phase 1), all cases of SARS-CoV-2 infection, regardless of severity, were admitted to isolation and treatment units. When this capacity was reached (approximately August-December 2020, a period with more community-transmission; study phase 2), admission was reserved for patients with or considered at risk for severe infection. ${ }^{5}$

Throughout admission, all management decisions were made by hospital clinicians independent of the study team and were informed by national COVID-19 treatment guidelines. ${ }^{3,4}$ The study team recorded management details until discharge, transfer to another health facility, or death.

The primary outcome of this study was a diagnosis of severe COVID-19. Based on criteria established by WHO, we considered patients to have severe COVID-19 if they fulfilled at least one of the following criteria during admission: 1) oxygen saturation $<90 \%$ on room air, 2) respiratory rate $>$ 30 breaths/minute, 3) showed signs of respiratory distress (chest in-drawing, nasal flaring, or grunting respirations), or 4) received oxygen therapy. ${ }^{6}$ Secondary outcomes included 
a composite measure of in-hospital outcome (death in-hospital or transfer to Uganda's national referral hospital due to progressive illness severity) and functional status at discharge, measured using Lansky or Karnofsky Performance Status.

Given differences in admission criteria across the two study phases, we performed our analyses in the general study population (primary analysis) and stratified by phase (secondary analysis). Continuous variables were expressed as medians (interquartile range), and categorical variables were summarized as frequencies and percentages with 95\% Cls provided for outcome data. In the primary analysis, factors associated with severe (versus mild-moderate) COVID-19 were determined using Wilcoxon rank sum, Fisher's exact, or $\chi^{2}$ tests, with two-sided $P$ values $\leq 0.05$ considered significant. Analyses were performed using Stata (v16, StataCorp, College Station, TX).

Each enrolled participant, their surrogate, or parent/guardian provided written informed consent. The study was approved by ethics committees at Columbia University, Uganda Virus Research Institute, and Uganda National Council for Science and Technology.

\section{RESULTS}

A total of 270 patients were enrolled during the study period (Supplemental Table 1; Supplemental Figure 1). The median age was 35 years (interquartile range: $27-43$ ); $83 \%$ were male. Cough, fever, and headache were the most common symptoms. Ten percent and 1\% had HIV and malaria coinfection, respectively. None of the patients tested had TB coinfection.

One hundred forty-six (54\%) and 124 (46\%) patients were admitted during study phases 1 and 2 , respectively (Table 1). Patients admitted during phase 1 were more likely to be male and to have recently traveled (Figure $1 \mathrm{~A}$ ). Patients admitted during phase 2 were more likely to be healthcare workers and to have hypertension and presented later in their illness course.

In the general study population, $18 \%(95 \% \mathrm{Cl}$ : 13-23) of patients met the primary outcome of WHO-defined severe COVID-19 (Supplemental Table 1). This included 5\% (95\% $\mathrm{Cl}: 2-10)$ and $33 \%(95 \% \mathrm{Cl}: 25-42)$ of patients admitted during study phases 1 and 2, respectively (Table 1, Figure 1B). In the general population, severe COVID-19 was significantly

TABLE 1

Characteristics of enrolled patients stratified by study phase

\begin{tabular}{|c|c|c|c|}
\hline Patient characteristic & Phase $1(N=146)$ & Phase $2(N=124)$ & $P$ value $^{*}$ \\
\hline Male sex, $n(\%)$ & $132 / 146(90)$ & $93 / 124(75)$ & 0.001 \\
\hline Age, years, median (IQR) & $34(27-42)$ & $36(28-46)$ & 0.332 \\
\hline Healthcare or laboratory worker, $n(\%)$ & $0 / 146(0)$ & $10 / 124(8)$ & $<0.001$ \\
\hline Travel within 14 days prior to admission, $n(\%)$ & 134/146 (92) & $27 / 124$ (22) & $<0.001$ \\
\hline Co-existing hypertension, $n(\%)$ & $3 / 146(2)$ & 22/124 (18) & $<0.001$ \\
\hline \multicolumn{4}{|l|}{ Symptoms reported, $n(\%)$} \\
\hline Cough & $36 / 146(25)$ & $74 / 124(60)$ & $<0.001$ \\
\hline Fever & $34 / 146(23)$ & 44/124 (35) & 0.028 \\
\hline Headache & $23 / 146(16)$ & $39 / 124(31)$ & 0.002 \\
\hline Rhinorrhea & 28/146 (19) & $32 / 124(26)$ & 0.190 \\
\hline Shortness of breath & $2 / 146(1)$ & $34 / 124(27)$ & $<0.001$ \\
\hline Sore throat & 12/146 (8) & $17 / 124(14)$ & 0.150 \\
\hline Diarrhea & 4/146 (3) & $7 / 124(6)$ & 0.355 \\
\hline Mildly symptomatic or asymptomatic, $n(\%)$ & $66 / 146(45)$ & 23/124 (19) & $<0.001$ \\
\hline Duration of illness prior to hospitalization, days, median (IQR)† & $4(2-5)$ & $6(3-7)$ & 0.026 \\
\hline \multicolumn{4}{|l|}{ Vital signs } \\
\hline Temperature $\geq 38^{\circ} \mathrm{C}, n(\%)$ & $8 / 146(5)$ & $7 / 124(6)$ & 0.953 \\
\hline Heart rate, beats/min, median (IQR) & $80(73-90)$ & $90(81-102)$ & $<0.001$ \\
\hline Respiratory rate, breaths/min, median (IQR) $\ddagger$ & $18(18-20)$ & $18(18-22)$ & 0.448 \\
\hline Systolic blood pressure, $\mathrm{mm}$ of $\mathrm{Hg}$, median (IQR) & $120(110-129)$ & $124(116-133)$ & 0.001 \\
\hline Oxygen saturation, \%, median (IQR) & $97(96-98)$ & $97(95-98)$ & 0.284 \\
\hline Glasgow Coma Score, median (IQR) & $15(14-15)$ & $15(14-15)$ & 0.025 \\
\hline Unable to ambulate without assistance, $n(\%)$ & $0 / 146(0)$ & $11 / 124(9)$ & $<0.001$ \\
\hline Mid-upper arm circumference, mm, median (IQR)§ & $20(18-23)$ & $22(20-24)$ & $<0.001$ \\
\hline \multicolumn{4}{|l|}{ Coinfections, $n(\%)$} \\
\hline Malaria & $3 / 146(2)$ & $1 / 124(1)$ & 0.697 \\
\hline HIV & $18 / 146(12)$ & $8 / 124(6)$ & 0.103 \\
\hline Microbiological TB\| & $0 / 16(0)$ & $0 / 5(0)$ & - \\
\hline \multicolumn{4}{|l|}{ Clinical management, $n(\%)$} \\
\hline Received oxygen therapy & $0 / 146(0)$ & $26 / 124(21)$ & $<0.001$ \\
\hline Received antibacterial agent & $75 / 146(51)$ & $96 / 124(77)$ & $<0.001$ \\
\hline Received HCQ or chloroquine & 28/146 (19) & $0 / 124(0)$ & $<0.001$ \\
\hline Received corticosteroids & $0 / 146(0)$ & $38 / 124(31)$ & $<0.001$ \\
\hline \multicolumn{4}{|l|}{ Patient outcomes, $n$ (\%) } \\
\hline Severe COVID-19 & $7 / 146(5)$ & $41 / 124(33)$ & $<0.001$ \\
\hline Died in-hospital & $0 / 146(0)$ & $0 / 124(0)$ & - \\
\hline Transferred to national referral hospital & $0 / 146(0)$ & $1 / 124(0.8)$ & 0.459 \\
\hline Karnofsky or Lansky score $\geq 80$ at discharge & $143 / 143(100)$ & $123 / 124(99)$ & 0.464 \\
\hline
\end{tabular}

$\mathrm{HCQ}=$ hydroxychloroquine; IQR = interquartile range, $\mathrm{TB}$ = tuberculosis.

*Wilcoxon rank-sum, Fisher's exact or $\chi^{2}$ test.

† Known for 135 patients.

‡ Known for 255 patients.

$\S$ Known for 214 patients.

\| Denominator of patients who underwent TB testing (urine TB-LAM or sputum Xpert MTB/RIF Ultra or smear)

IDefined as absence of reported cough, fever, headache, rhinorrhea, shortness of breath, sore throat, diarrhea, or night sweats. 

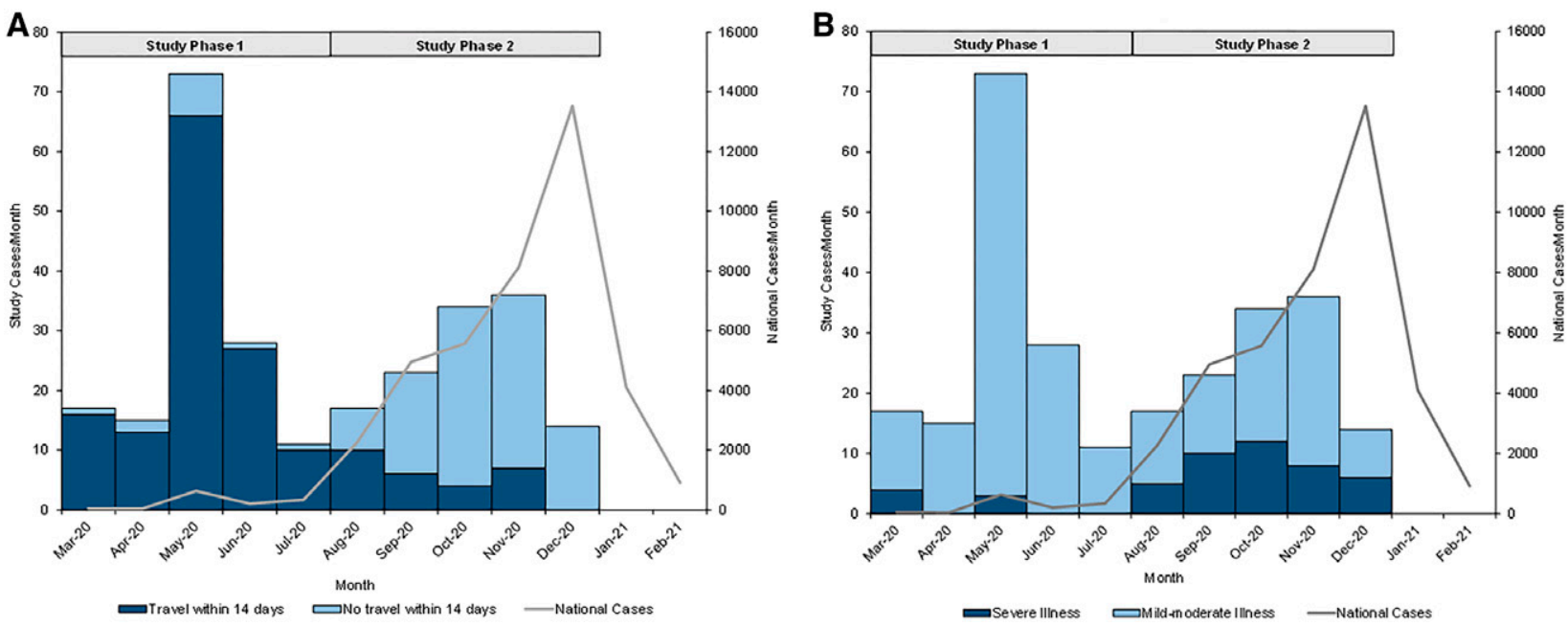

FIGURE 1. Epidemiologic curve of enrolled patients, stratified by (A) travel history and (B) illness severity and plotted with national COVID-19 case counts. National COVID-19 case counts were obtained from the WHO COVID-19 Uganda Dashboard. ${ }^{7}$ Study phase 1: March-July 2020; study phase 2: August-December 2020. This figure appears in color at www.ajtmh.org.

associated with older age, male sex, and a longer duration of illness prior to admission (Table 2). These associations were driven by patients admitted during phase 2 (Supplemental Tables 2 and 3). Across and within each phase, we observed no significant associations between illness severity and HIV coinfection. One patient in phase 2 required transfer to the national referral hospital; no patients died in-hospital (Table 1).

During admission, $10 \%$ of patients received oxygen therapy, delivered via concentrators and cylinders, including $21 \%$ of patients admitted during phase 2 (Table 1, Supplemental Table 1). Patients admitted during phase 1 were more likely to receive

TABLE 2

Characteristics of patients with and without severe COVID-19 across both study phases

\begin{tabular}{|c|c|c|c|}
\hline Patient characteristic & Severe illness $(N=48)$ & Mild-moderate illness $(N=222)$ & $P$ value ${ }^{*}$ \\
\hline Male sex, $n(\%)$ & $45 / 48(94)$ & $180 / 222(81)$ & 0.033 \\
\hline Age, years, median (IQR) & $41(35-50)$ & $33(27-41)$ & 0.0001 \\
\hline Co-existing hypertension, $n(\%)$ & $5 / 48(10)$ & 20/222 (9) & 0.784 \\
\hline \multicolumn{4}{|l|}{ Symptoms reported, $n(\%)$} \\
\hline Cough & $33 / 48(69)$ & 77/222 (35) & $<0.0001$ \\
\hline Fever & $27 / 48(56)$ & $51 / 222(23)$ & $<0.0001$ \\
\hline Headache & 9/48 (19) & $53 / 222(24)$ & 0.444 \\
\hline Rhinorrhea & $4 / 48(8)$ & $56 / 222(25)$ & 0.011 \\
\hline Shortness of breath & $22 / 48(46)$ & $14 / 222(6)$ & $<0.0001$ \\
\hline Sore throat & $6 / 48(13)$ & 23/222 (10) & 0.664 \\
\hline Diarrhea & $3 / 48(6)$ & $8 / 222(4)$ & 0.419 \\
\hline Duration of illness prior to hospitalization, days, median (IQR)† & $6(3-7)$ & $4(2-7)$ & 0.009 \\
\hline \multicolumn{4}{|l|}{ Vital signs } \\
\hline Temperature $\geq 38^{\circ} \mathrm{C}, n(\%)$ & $7 / 48(15)$ & $8 / 222(4)$ & 0.008 \\
\hline Heart rate, beats/min, median (IQR) & $91(79-104)$ & $83(76-93)$ & 0.002 \\
\hline Respiratory rate, breaths/min, median (IQR)‡ & $22(19-24)$ & $18(18-20)$ & - \\
\hline Systolic blood pressure, mm Hg, median (IQR) & $126(112-132)$ & $120(114-130)$ & 0.373 \\
\hline Oxygen saturation, $\%$, median (IQR) & $95(92-98)$ & $97(96-98)$ & - \\
\hline Glasgow Coma Score, median (IQR) & $15(14-15)$ & $15(14-15)$ & 0.189 \\
\hline Unable to ambulate without assistance, $n(\%)$ & $10 / 48(21)$ & $1 / 222(0.5)$ & $<0.0001$ \\
\hline Mid-upper arm circumference, mm, median (IQR)§ & $22(20-24)$ & $21(19-23)$ & 0.309 \\
\hline \multicolumn{4}{|l|}{ Coinfections, $n(\%)$} \\
\hline Malaria & $0 / 48(0)$ & $4 / 222(2)$ & - \\
\hline HIV & $5 / 48(10)$ & 21/222 (9) & 0.791 \\
\hline Microbiological TB $\|$ & $0 / 2(0)$ & $0 / 19(0)$ & - \\
\hline \multicolumn{4}{|l|}{ Clinical management, $n(\%)$} \\
\hline Received oxygen therapy & $26 / 48(54)$ & $0 / 222(0)$ & - \\
\hline Received antibacterial agent & 40/48 (83) & $131 / 222(59)$ & 0.002 \\
\hline Received $\mathrm{HCQ}$ or chloroquine & $2 / 48(4)$ & $26 / 222(12)$ & 0.189 \\
\hline Received corticosteroids & $26 / 48(54)$ & $12 / 222(5)$ & $<0.0001$ \\
\hline
\end{tabular}

$\mathrm{HCQ}=$ hydroxychloroquine; IQR = interquartile range; TB = tuberculosis.

*Wilcoxon rank-sum, Fisher's exact, or $\chi^{2}$ test.

† Known for 135 patients.

$\ddagger$ Known for 255 patients.

§Known for 214 patients.

|| Denominator of patients who underwent TB testing (urine TB-LAM or sputum Xpert MTB/RIF Ultra or smear). 
hydroxychloroquine or chloroquine, whereas those admitted during phase 2 were more likely to receive corticosteroids. Among patients with severe illness admitted during phase 2, those who received corticosteroids were older and had more deranged vital signs (Supplemental Table 4). Nearly all patients administered corticosteroids received oxygen therapy and antibacterial agents.

\section{DISCUSSION}

Among a prospective cohort of patients admitted to a national COVID-19 treatment unit in Uganda during the first two phases of the epidemic, the incidence of WHO-defined severe COVID-19 ranged from $5 \%$ to $33 \%$. Consistent with data from high-income countries, older age and male sex were associated with illness severity.8,9 Although HIV and TB coinfections have been associated with COVID-19-related mortality elsewhere in the region, ${ }^{10}$ these coinfections were infrequent in our cohort, as was malaria. Similar to a recent study from Malawi, we observed no significant association between HIV coinfection and illness severity. ${ }^{11}$ Given these likely context-specific findings, continued efforts are needed to elucidate the epidemiology of severe COVID-19 in SSA.

During the peak epidemic phase, over $20 \%$ of patients received oxygen therapy and $63 \%$ with severe illness received corticosteroids. Because administration of oxygen is an essential treatment of all severe respiratory infections, expanded access to oxygen therapy in SSA is a global imperative. ${ }^{12}$ In the interim, efforts are needed to optimize targeted delivery of oxygen therapy, corticosteroids, and other evidence-based treatments to severely ill patients in the region.

Despite substantial incidence of severe COVID-19 during the epidemic peak, no patients died in-hospital, and only one was transferred to the national referral hospital due to progressive illness severity. This finding is largely consistent with data reported across Uganda, where the national case fatality rate for SARS-CoV-2 infection during the study period was $0.7 \% .{ }^{1}$ Although under-reporting is possible and reasons for low mortality remain uncertain, a relatively young population, lower prevalence of high-risk comorbidities, and meteorological variables have been proposed. ${ }^{13,14}$ Nonetheless, as COVID-19 incidence and mortality in SSA are rising substantially, improvements in vaccine deployment and acute care capacity remain urgently needed to avoid preventable mortality. $^{15}$

This study has limitations. First, our findings were derived from a single-center study. Second, the frequency of HIV, $\mathrm{TB}$, and malaria was low, under-powering comparisons between these coinfections and outcomes. Third, our protocol excluded pregnant women, who are known to have higher risk of severe COVID-19. ${ }^{16}$ Fourth, we only obtained in-hospital outcomes. Although nearly all patients were discharged alive with normal functional status, future studies are needed to characterize longer-term outcomes and functional sequelae of COVID-19 in SSA.

Despite low mortality, hospital incidence of severe COVID19 during the first epidemic peak in Uganda was substantial. Improvements in vaccine deployment and acute care capacity are urgently needed to prevent and manage severe COVID-19 in SSA.
Received May 17, 2021. Accepted for publication June 10, 2021.

Published online August 9, 2021.

Note: Supplemental materials appear at www.ajtmh.org.

Acknowledgments: We thank the patients enrolled in this study as well as our fellow clinicians and scientists for providing outstanding clinical care and laboratory work despite considerable personal risk. Members of the Collaboration for Clinical and Laboratory Characterization of COVID-19 in Uganda are as follows: National Influenza Center, Department of Arbovirology, Emerging and Re-emerging Infectious Diseases, Uganda Virus Research Institute, Entebbe, Uganda (Barnabas Bakamutumaho, Nicholas Owor, John Kayiwa, Joyce Namulondo, Timothy Byaruhanga, Julius J. Lutwama, Joweria Nakaseegu, Jocelyn Kiconco, Annet Nankya, Irene Ataliba, Samuel Wavamuno); Viral Hemorrhagic Fever Program, Uganda Virus Research Institute, Entebbe, Uganda (Stephen Balinandi, Luke Nyakaruhuka, Jimmy Baluku, Alex Tumusiime, Jackson Kyondo, Sophia Mulei, Kilama Kamugisha, Gloria Akurut, Diana Nahamya); Immunisable Diseases Unit/Expanded Programme on Immunisation Laboratory, Uganda Virus Research Institute, Entebbe, Uganda (Peter Eliku, Phiona Tushabe, Joshua Buule, Joseph Gaizi, Prossy Namuwulya, Arnold Mugaga, Mary Nyacho, Henry Bukenya, Josephine Bwogi); European and Developing Countries Clinical Trials Partnership-Eastern Africa Consortium for Clinical Research, Uganda Virus Research Institute, Entebbe, Uganda (Bernard Kikaire); Entebbe Regional Referral Hospital, Entebbe, Uganda (Moses Muwanga, Christopher Nsereko, Emmanuel Rwamutwe, Roselyn Mutonyi, Josephine Achan, Lucy Wanyenze, Alice Ndazarwe, Ruth Nakanjako, Richard Natuhwera, Annet Nsangi); Uganda Peoples' Defence Force, Kampala, Uganda (Henry Kyobe Bosa); Public Health Emergency Operations Centre, Ministry of Health, Kampala, Uganda (Felix Ocom); Makerere University School of Public Health, Kampala, Uganda (James Arinaitwe); Division of Pulmonary, Allergy, and Critical Care Medicine, Columbia University Irving Medical Center, New York, USA (Matthew J. Cummings, Max R. O'Donnell). The American Society of Tropical Medicine and Hygiene has waived the Open Access fee for this article due to the ongoing COVID-19 pandemic.

Financial support: This work was supported by the National Center for Advancing Translational Sciences (UL1TR001873 to Columbia University, sub-award to M.R.O.), the National Institute of Allergy and Infectious Diseases (F32Al147528 to M.J.C.), and the MakCHSBerkeley-Yale Pulmonary Complications of AIDS Research Training (PART) Program (D43TW009607, sub-award to B.B.) from the Fogarty International Center, National Institutes of Health. Additional support was provided through the DELTAS Africa Initiative (grant no. 107743). The DELTAS Africa Initiative is an independent funding scheme of the African Academy of Sciences, Alliance for Accelerating Excellence in Science in Africa, and is supported by the New Partnership for Africa's Development Planning and Coordinating Agency with funding from the Wellcome Trust (grant no. 107743) and the UK Government. The funders had no role in study design, data collection, analysis or interpretation, manuscript preparation, or decision to publish.

Disclosure: M.J.C and M.R.O. were investigators for clinical trials evaluating the efficacy and safety of remdesivir, convalescent plasma, and anti-SARS-CoV-2 hyperimmune globulin in hospitalized patients with COVID-19, sponsored by Gilead Sciences, Amazon, Inc., and the U.S. National Institutes of Health, respectively. Compensation for this work was paid to Columbia University. The remaining authors declare no conflicts of interest.

Authors' addresses: Barnabas Bakamutumaho, Nicholas Owor, John Kayiwa, Joyce Namulondo, and Timothy Byaruhanga, Uganda Virus Research Institute, Entebbe, Uganda, E-mails: bbarnabas2001@ yahoo.com, nicowor@gmail.com, jkayiwa@yahoo.com, jonacla.j@ gmail.com, and tssekandi@gmail.com. Matthew J. Cummings, Columbia University Medical Center, New York, New York, E-mail: mjc2244@columbia.edu. Moses Muwanga, Christopher Nsereko, Emmanuel Rwamutwe, Roselyn Mutonyi, Josephine Achan, Lucy Wanyenze, Alice Ndazarwe, Ruth Nakanjako, Richard Natuhwera, and Annet Nsangi, Entebbe Regional Referral Hospital, Entebbe, Uganda, E-mails: docmuwanga@yahoo.com, chrisdoc23@yahoo. com, rwemla15@gmail.com, mutonyiroselyn@gmail.com, josephinegladys2015@gmail.com, Wanyenzelucy2005@gmail.com, alicendazarwe2019@gmail.com, nakanjakoruth164@gmail.com, natuhwerarichard@gmail.com, and nsangiannet@gmail.com. Henry 
Kyobe Bosa, Uganda People's Defence Force, Kampala, Uganda, E-mail: hskyobe@gmail.com. Felix Ocom, Ministry of Health, Kampala, Uganda, E-mail: felixocom@gmail.com. Max R. O'Donnell, Columbia University Medical Center, New York, New York, E-mail: mo2130@columbia.edu. Bernard Kikaire and Julius J. Lutwama, Uganda Virus Research Institute, Entebbe, Uganda, E-mails: bkikaire@uvri.go.ug and jlutwama03@yahoo.com.

This is an open-access article distributed under the terms of the Creative Commons Attribution (CC-BY) License, which permits unrestricted use, distribution, and reproduction in any medium, provided the original author and source are credited.

\section{REFERENCES}

1. Africa Centres for Disease Control and Prevention, 2021. Africa CDC Covid-19 Dashboard. Available at: https://africacdc.org/ covid-19/. Accessed July 14, 2021.

2. Rice BL et al., 2021. Variation in SARS-CoV-2 outbreaks across sub-Saharan Africa. Nat Med 27: 447-453.

3. Kirenga B et al., 2020. Characteristics and outcomes of admitted patients infected with SARS-CoV-2 in Uganda. BMJ Open Respir Res 7: e000646.

4. World Health Organization, 2021. A High-Level Overview of Emerging Issues in Each Phase of the Pandemic. Available at: https://www.who.int/docs/default-source/coronaviruse/riskcomms-updates/update51_pandemic_overview_where_are we_now.pdf?sfvrsn=709278aa_5. Accessed May 14, 2021.

5. Ministry of Health, 2021. National Guidelines for Management of COVID-19. Available at: https://www.health.go.ug/cause/ national-guidelines-for-management-of-covid-19/. Accessed May 14, 2021.

6. Siemieniuk R, et al., 2020. A living $\mathrm{WHO}$ guideline on drugs for covid-19. BMJ 370: m3379.
7. World Health Organization, 2021. Coronavirus (COVID-19) Dashboard, Uganda. Available at: https://covid19.who.int/region/ afro/country/ug. Accessed May 14, 2021

8. Peckham H, de Gruijter NM, Raine C, Radziszewska A, Ciurtin C, Wedderburn LR, Rosser EC, Webb K, Deakin CT, 2020. Male sex identified by global COVID-19 meta-analysis as a risk factor for death and ITU admission. Nat Commun 11: 6317.

9. O'Driscoll M, Ribeiro Dos Santos G, Wang L, Cummings DAT, Azman AS, Paireau J, Fontanet A, Cauchemez S, Salje $H$, 2021. Age-specific mortality and immunity patterns of SARSCoV-2. Nature 590: 140-145.

10. Vermooten B et al., 2020. Risk factors for COVID-19 death in a population cohort study from the Western Cape Province, South Africa. Clin Infect Dis August 29: ciaa1198.

11. Morton B et al., 2021. Distinct clinical and immunological profiles of patients with evidence of SARS-CoV-2 infection in subSaharan Africa. Nat Commun 12: 3554.

12. Nakkazi E, 2021. Oxygen supplies and COVID-19 mortality in Africa. Lancet Respir Med 9: e39.

13. Njenga MK, Dawa J, Nanyingi M, Gachohi J, Ngere I, Letko M, Otieno CF, Gunn BM, Osoro E, 2020. Why is there low morbidity and mortality of COVID-19 in Africa? Am J Trop Med Hyg 103: 564-569.

14. Lawal Y, 2021. Africa's low COVID-19 mortality rate: a paradox? Int J Infect Dis 102: 118-122.

15. Africa Centres for Disease Control and Prevention, 2021. New SARS-CoV-2 Variants in Africa. Available at: https://africacdc. org/download/new-sars-cov-2-variants-in-africal. Accessed May 14, 2021.

16. Allotey J, et al., 202. Clinical manifestations, risk factors, and maternal and perinatal outcomes of coronavirus disease 2019 in pregnancy: living systematic review and meta-analysis $B M J$ 370: $\mathrm{m} 3320$. 